\title{
Design of Feeder Terminal Communication Based on IEC61850 Standard
}

\section{Tongtong-Sun ${ }^{1}$, Limin-Huo², Zhenyue-Yan ${ }^{3}$, Zu-Zhang ${ }^{4}$}

\author{
${ }^{1}$ College of Mechanical and Electrical Engineering, Agricultural University of Hebei, Hebei Baoding, 071001
}

\begin{abstract}
In this paper, IEC61850 is introduced into the feeder system, which can solve the problem of information sharing and interoperability between different distribution terminals. In order to realize the real-time interaction of information, the information function model of feeder terminal is constructed by using the logical node defined by IEC61850, and the information exchange service used in the terminal is mapped to the corresponding communication mode. Where the client / server model is mapped to the manufacturing message specification MMS and the GOOSE messages are mapped to the IOS Ethernet data link layer. So that each switch in the distribution network feeder realizes the point-to-multipoint communication and the interoperation between different FTUs. Finally, we build an experimental platform to verify the mapping method to meet the requirements.
\end{abstract}

Keywords: IEC61850, GOOSE, SCL, MMS manufacturing message specification, Interoperability

\section{INTRODUCTION}

Distribution automation system has a large number of distribution terminals. A variety of users in the choice of different terminals according to demand, but also brought a series of problems. For example, the different vendors can not interoperate between devices, and the realization of the functions and data interfaces used are not the same.

The IEC61850 standard is proposed, which solves the problem that the different devices can not operate mutually, and the functions are different. It defines a large number of data types and logical nodes. And it covering almost all areas of distribution automation system, which uses a unified model and a unified service interface makes the same network to achieve interoperability between the different devices ${ }^{[1]}$.A second reference analyzes plug-and-play techniques for distribution terminals. The third reference proposes the use of WebServices + IEC60870-5-104 in the distribution network to transmit real-time data mapping.In literature four, the mapping method of applying IEC61850 to distribution network is studied.

The above research is only concerned with the information transmission between the main station and distribution terminal. When the feeder is in trouble, there is little research on how to realize the interoperation quickly between the terminals. Based on the existing research. In this paper, we propose a fast message GOOSE mapping to IOS Ethernet to realize the interoperability between the terminals

\section{INFORMATION MODEL OF FEERDER}

\section{TERMINAL}

IEC61850 uses the object-oriented modeling method to carry on the modeling to the secondary equipment of the feeder automation system. In order to meet the function 
of the free distribution and distribution. It decomposes all functions into the logical nodes, and the logical nodes are connected by the logical connections, which are used to realize data exchange ${ }^{[5]}$. IEC61850 defines a large number of classes and each class has a specific attribute and the corresponding access service. The user can derive the required instances from the appropriate classes as needed. Each instance inherits the attributes and service properties of the class ${ }^{[6]}$.As shown in Figure 1, according to the IEC61850 information transmission characteristics of the feeder terminal FTU can be defined as three logical devices, they are control module LD1, protection module LD2 and power module LD3. Each logical device contains LLNO and the LPHD nodes. The LPHD models the common information of the physical devices, such as nameplate information, device self-test results and so on ${ }^{[7]}$. The logical node LLNO contains the information about the physical device, controls the self-test of the intelligent electronic device and so on. The GGIO in LD1 is a general-purpose process logic node, which can be used to access analog or state quantities that are not easily modeled, such as switch-controlled soft platen information. When an overcurrent fault occurs in the system, the PTOC controls the CSWI (the switch controller) of LD1.

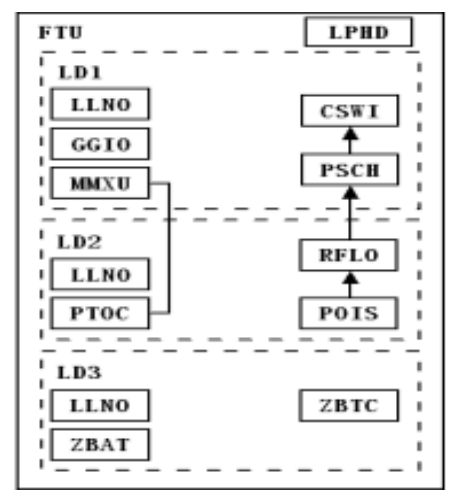

Fig. 1 information mode of FTU

Of course, the monitoring center can also control the CSWI to achieve the remote control function. The PDIS (the distance protection) and RFLO (the fault location) can identify the fault point in the system in real time, and use the PSCH (the protection scheme) generation solution to control the CSWI (the switch controller) action. The LD3 with power management includes ZBAT (battery) and ZBTC (battery charge management) two logical nodes to ensure the continued normal operation of the terminal.

\section{FTU information sharing and interoperability}

According to the IEC61850 standard, the information exchange service used by the distribution terminal is mapped to the corresponding communication mode $\mathrm{e}^{[8]}$. Where the client / server model is mapped to the Manufacturing Message

Specification (MMS), and the GOOSE (Generalized Substation Event) message is mapped to the IOS Ethernet data link layer with reference to the IEC 61850 standard.

\subsection{Information exchange}

\subsubsection{SCL configuration file}

Based on the IEC61850 standard feeder automation terminal communication model, there is a logic device D1 in the FTU, which it contains three logical nodes: LLNO, the logical physical device LPHD and the measurement logical node MMXU. The XML files of each logical node are written and used to represent the data and attributes of the logical nodes. According to the hierarchical characteristics of the XML files, the Substation Configuration Language (SCL) is used to describe the MMS objects. Which is automatic generation of MMS types object space.

\subsubsection{MMS services and mapping}

MMS is an open system interconnection (OSI) application layer of a protocol standard, it is mainly used in the transmission of the control information between the production equipment. IEC61850-8-1 defines the mapping of the client / server model to MMS in detail. Specifically, the servers in the IEC61850 are mapped to the VMDs of the MMS, and Logical Devices map to the Domain Objects.

In order to achieve the information sharing between the terminals, the FTU internal information flow shown in 
Figure 2. The IEC61850-8-1 information model and the service model through the abstract communication service interface ACSI mapping to MMS, and then uploaded to the Ethernet ${ }^{[9]}$, within the same subnet FTU can be obtained from the Ethernet real-time information and simultaneously upload the machine data. The implementation of MMS generally uses SISCO MMS-EASE Lite software. It is the MMS application programming interface and supports each kind of popular network environment.

\subsection{Interoperability implementation}

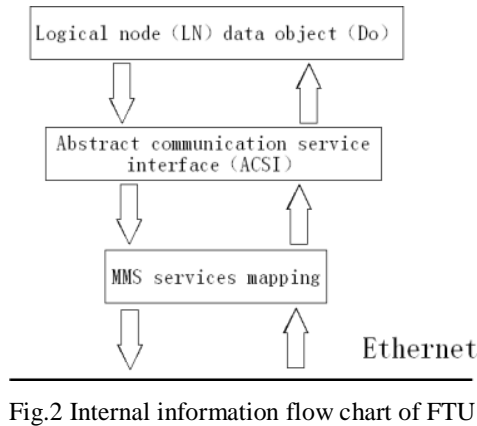

The network sharing of data information between terminals is described above. However, when a fault occurs in the feeder automation system, the GOOSE model can be used to send control commands to allow for the removal of faults between the devices.

\subsubsection{GOOSE transmission model}

GOOSE transmission model is divided into four layers. They are the application layer, presentation layer, data link layer and physical layer. In the application layer, the mapping of GOOSE and the service object produce the application protocol data unit APDU, and generate the GOOSE message ${ }^{[10]}$. At the presentation layer, GOOSE encodes the basic coding rule (BER) of ASN.1 with abstract syntax. GOOSE maps the code directly to the data link layer.

\subsubsection{GOOSE message sending}

GOOSE message in the design of the sending process shown in Figure 3.

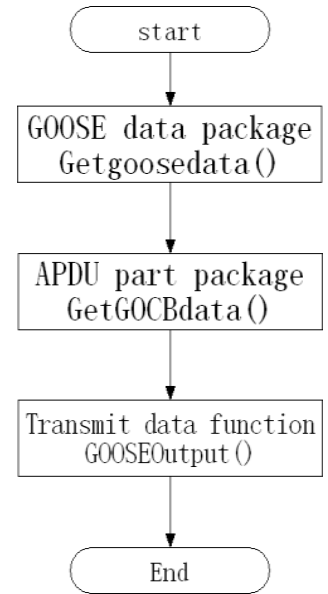

Fig.3 Flowchart of GOOSE message sending

First, the data portion of the GOOSE message is encapsulated by the function Getgoosedata (), which encapsulates the GOOSE data content in the subscription data set ${ }^{[11]}$ and declares as follows: Getgoosedata(Uint16*len,Uint8dst[])

The function contains two parameters, the first parameter is GOOSE data byte length and the second parameter is the stored GOOSE data array. Then the function GetGOCBdata () will be part of the GOOSE APDU package,including GocbRef, GoID, DataSet and other attributes. The statement of the modified function is as follows: GetGOCBdata(Uint8*buffer,Uint16*len)

The final packets of the GOOSE sent through the Ethernet. So, GOOSEOutput () eventually need to call GetGOCBdata (Uint8 *buffer, Uint16 *len) this FTU using the send data function. This function contains three parameters, the first parameter is the Socket port number used by sending GOOSE packets need to specify the port, the second parameter is the data to be transmitted is stored in buf and the third parameter is the length of data bytes to be sent ${ }^{[12]}$.

\section{FTU system design}

\subsection{Hardware equipment diagram}

The terminal of this project is embedded cmputer control system terminal. It is suitable for outdoor harsh operating environment, and has high reliability. In order to satisfy the functional requirements and the later function expansion, this design will use the ST company 
STM32F303 (ARM Cortex-M4 kernel) as the main control chip. The ARMCortex -M4 processor is developed based on the Cortex-M3 kernel. Its performance is improved by $20 \%$ compared with Cortex-M3 and increased floating-point, DSP, parallel computing and so on.

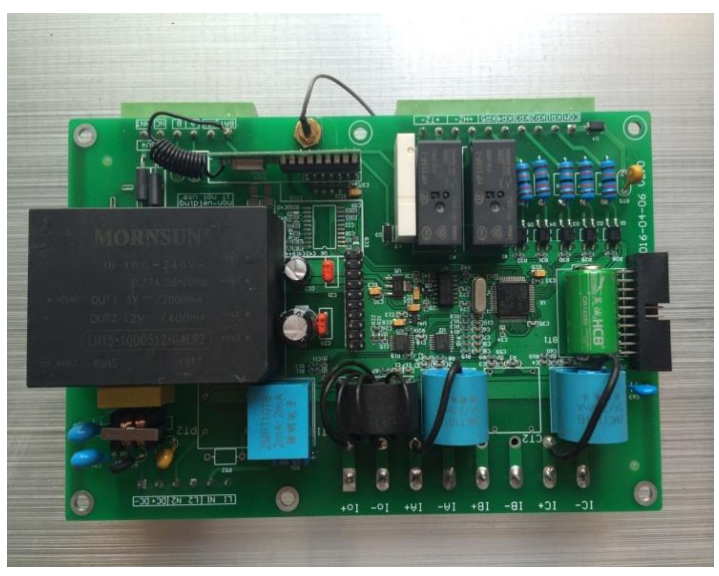

Fig.4 CPU hardware equipment diagram of FTU

Details as shown in Figure 4, the integrated circuit of the processormainly includes: CPU, DSP, FPU and two serial communication port, current / voltage transformer, DMA controller, interrupt controller, USB, QSPI, SDRAM and Ethernet controller. It has a DC analog acquisition and Ethernet, UART, USB and other communication functions, which to provide device operation status indicator (RUN) and reception indicator, CAN data transmission and reception indicator, the network data reception and other operating status indication.

\subsection{FTU internal program flow chart}

As shown in Figure 5, this is the feeder terminal server-side program flow chart.

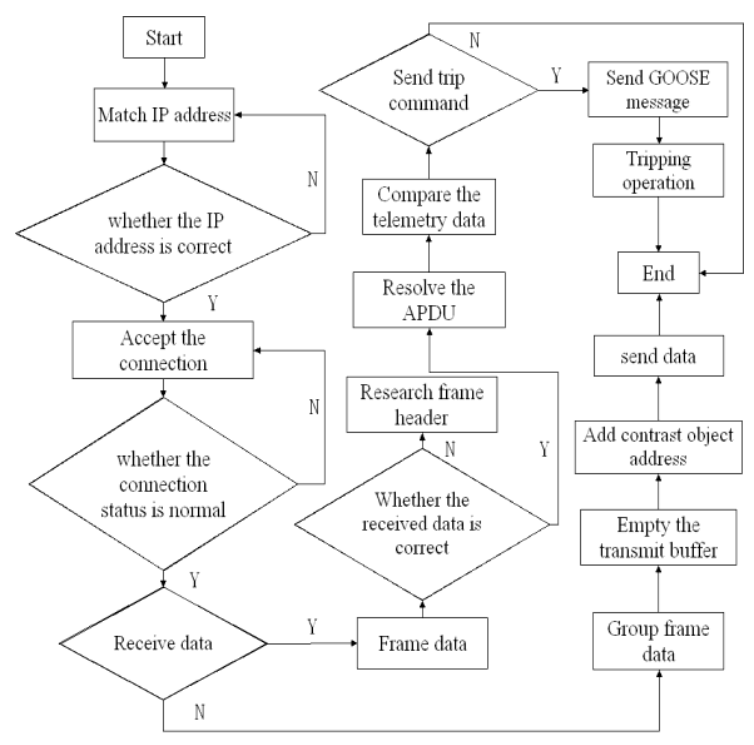

Fig.5 program flow chart of FTU

After the system starts, it will create a peer to peer communication task with a function. The priority of the task is 130 and the heap size is 1024 bytes. The server program is running, the system will determine whether the server is in the listening state. whether the socket socket is in the listen state, if not in the listening state, the system create a socket and activate the server listening state.

At the same time, it is determined whether the IP address of the connection from the neighboring client is IP in the server list, so that it can shield the active connection from the illegal client. It will accept the connection from the client. The system will determine the link status and use the recv () function to determine whether new data is being received.

If the system receives the APDU part of the complete GOOSE message, then enter the solution meal program. First, the system judges whether the received frame header is $68 \mathrm{H}$, then judge whether the received APCI length is 6 bytes, finally, the system determines whether the length of APDU in the received APCI data is greater than 253. If the three conditions are all through then the system is running and compare the current, voltage and other data from the local or Ethernet, and to determine whether a fault and issue a trip command. Otherwise, the frame header information is searched again. 
If the system have not received data into the framing process, the group procedure first determines whether the data link layer is normal in the GOOSE transport model. Then empty the transmit buffer and initializes the transmit buffer data pointer. The system determines whether to send out the alarm command, and use the send () function to send the current information in real time.

\subsection{Set up experimental model}

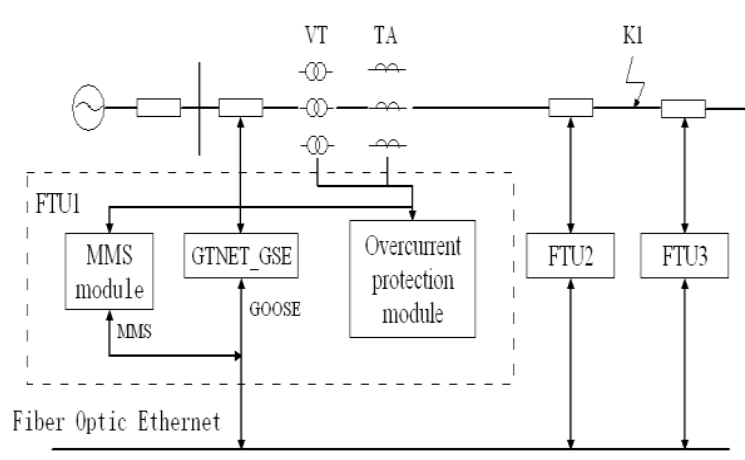

Fig.6 Flow chart of function module between different terminals

In order to verify the feasibility of information sharing and interoperation between FTUs proposed in this paper, and set up the test platform as shown in Figure 6. It includes the protection of lines and three distribution terminals, and all devices are configured with IP addresses for the same field and communicate in real-time over fiber-optic Ethernet.

Among them, the current module of the FTU1 will collect the current signal of the current transformer, and to determine. Then GTNET_GSE will be over-current results in the form of GOOSE packets sent to the Ethernet. At the same time, the MMS module of the feeder terminal is responsible for transmitting telemetry, including current, voltage and feeder power. When the fault occurs in the power system K1, FTU1 and FTU2 over-current module are detected over the line and the network through the GTNET_GSE network to send GOOSE message. The adjacent FTU 3 receives the fault message from the Ethernet and sends the information to the network. Finally, FTU2 in the comparison point after the current size to determine their own point at the occurrence of short-circuit fault. And to sends a control command immediately to the circuit breaker under its control to trip.

\section{5 conclusion}

In order to improve power supply reliability of distribution network, and realization of intelligent distributed protection function of distribution terminal. In this paper, according to the IEC61850 standard and the functional requirements of the distribution terminal. In this paper, according to the IEC61850 standard and the functional requirements of the distribution terminal, and the information model of intelligent power distribution terminal is established and the mapping of IEC61850 and MMS is deeply studied, and realizing the information sharing between different FTUs .According to the principle of GOOSE differential protection to achieve the distribution terminal differential protection. Finally, the hardware circuit and software program are designed, and it is tested and analyzed. The design of intelligent distribution terminal can accurately judge the fault area. When the short circuit or ground fault occurs. The circuit breaker can cut off the fault point quickly, and restoring power to the faultless line, reduce the scope of the accident and shorten the accident time. It can promote the construction of intelligent distribution network.

\section{REFRENCES:}

[1] Hongwang-xin. Design of Micro-grid Adaptive Protection System Based on IEC61850[J]. Smart grid,2015,3(4):337-343.

[2] Huichao-wu,Wu yi,Haibing-zhu,Zhijun-tan,Mingxiang-liu. Research on plug and play system of distribution automation terminal based on IEC61850 standard [J]. Power supply,2015,01:60-63.

[3] Guozheng-han,Bingyin-xu,Jiale-suonan,Guoxian-lv,Jianmin-wu. Communication technology for distribution automation based on IEC $61850 \quad[\mathrm{~J}]$. Power Svstem Protection and Control,2013,41(2):62-66.

[4] Nhat Nguyen-Dinh,Gwan-Su Kim and Hong-Hee Lee.A study on GOOSE communication based on IEC61850 using MMS ease Lite[C]. Ulsan: International Conference on Control, 2007.

[5] Qilin-zhang,Xianpei-wang,Zhao yu. Analysis on Cascading Failures of Substation Automation System Based on IEC 61850 [J]. Automation of Electric Power Systems,2013,2(37):61-66. 
[6] Cuiran-zhao. Research on the Modeling of Distribution Terminal and its Self-Description Based on IEC 61850 [D].Baoding: School of Electrical\&Electric Engineer,2013(27,33-34).

[7] C.Hoga and P.Skare, IEC61850Projects in Germany and Switzerland,IEEE PES2006,Page(s):390-393.

[8] Jianwei-gu,Tiefeng-zhang,Shujuan-han. Study on Applications of Distribution Automation System Based on IEC61850 International Standards [J]. Electric Power Science and Engineering,2012,1(28):30-36.

[9] Wong G, Brand K P,Rudolph T.Interoperability testing and validation of IEC61850 protective relays.[C] Anais do International Protection Testing Symposium,2004.
[10] Kanjun-zhang,Yongjun-xia,Wang jin,Chen lei,Shu xin. GOOSE Communication Technology in Smart Substations [J]. Electric Power of Hubei, 2013,1(37):7-10

[11] Xinxi-yuan. The Application of IEC61850 in Smart Distributed Feeder Automation and Modeling of Distributed Terminal [D].Hefei: Hefei University Of Technology,2013(42).

[12] Changshou-zhao, Yuzhong-zhang. Design of point to point voice communication software based on UDP protocol [J]. Computer programming skills and maintenance. 2013,11(16):62-65. 